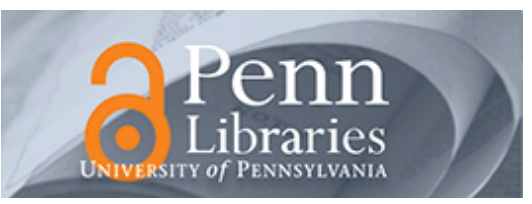

University of Pennsylvania

ScholarlyCommons

\title{
Evidence for Spring Loaded Inverted Pendulum Running in a Hexapod Robot
}

\author{
Richard Altendorfer \\ University of Michigan - Ann Arbor \\ Uluc Saranli \\ University of Michigan - Ann Arbor \\ Haldun Komsuoglu \\ University of Michigan - Ann Arbor \\ Daniel E. Koditschek \\ University of Pennsylvania, kod@seas.upenn.edu \\ H. Benjamin Brown Jr. \\ Carnegie Mellon University
}

Follow this and additional works at: https://repository.upenn.edu/ese_papers

See next page for additional authors

\section{Recommended Citation}

Richard Altendorfer, Uluc Saranli, Haldun Komsuoglu, Daniel E. Koditschek, H. Benjamin Brown Jr., Martin Buehler, Ned Moore, Dave McMordie, and Robert Full, "Evidence for Spring Loaded Inverted Pendulum Running in a Hexapod Robot", . January 2001.

\section{BibTeX entry}

@inproceedings $\{$ Altendorfer-Saranli-Komsuoglu.ISER2000, author = $\{$ Altendorfer, R. and Saranli, U. and Komsuoglu, H. and Koditschek, D. E. and Brown, Jr. H. B. and Buehler, M. and Moore, N. and McMordie, D. and Full, R.\}, title = \{Evidence for Spring Loaded Inverted Pendulum Running in a Hexapod Robot\}, booktitle = \{Proceedings on International Symposium on Experimental Robotics $\}$, year $=\{2000\}\}$

This work is supported by DARPA/SPAWAR under contract N66001-00-C-8026. We thank Rodger Kram and Claire Farley for the use of the force plateform and Irv Scher for collaboration at an early stage of this project.

This paper is posted at ScholarlyCommons. https://repository.upenn.edu/ese_papers/666

For more information, please contact repository@pobox.upenn.edu. 


\title{
Evidence for Spring Loaded Inverted Pendulum Running in a Hexapod Robot
}

\author{
Abstract \\ This paper presents the first evidence that the Spring Loaded Inverted Pendulum (SLIP) may be "anchored \\ " in our recently designed compliant leg hexapod robot, RHex. Experimentally measured RHex center of \\ mass trajectories are fit to the SLIP model and an analysis of the fitting error is performed. The fitting \\ results are corroborated by numerical simulations. The "anchoring " of SLIP dynamics in RHex offers \\ exciting possibilities for hierarchical control of hexapod robots. \\ For more information: Kod*Lab \\ Comments \\ BibTeX entry \\ @inproceedings $\{$ Altendorfer-Saranli-Komsuoglu.ISER2000, author $=\{$ Altendorfer, R. and Saranli, \\ U. and Komsuoglu, H. and Koditschek, D. E. and Brown, Jr. H. B. and Buehler, M. and Moore, N. \\ and McMordie, D. and Full, R. $\}$, title = \{Evidence for Spring Loaded Inverted Pendulum Running in \\ a Hexapod Robot $\}$, booktitle $=\{$ Proceedings on International Symposium on Experimental \\ Robotics $\}$, year $=\{2000\}\}$ \\ This work is supported by DARPA/SPAWAR under contract N66001-00-C-8026. \\ We thank Rodger Kram and Claire Farley for the use of the force plateform and \\ Irv Scher for collaboration at an early stage of this project. \\ Author(s) \\ Richard Altendorfer, Uluc Saranli, Haldun Komsuoglu, Daniel E. Koditschek, H. Benjamin Brown Jr., Martin \\ Buehler, Ned Moore, Dave McMordie, and Robert Full
}




\title{
Evidence for Spring Loaded Inverted Pendulum Running in a Hexapod Robot
}

\author{
Richard Altendorfer, Uluç Saranli, Haldun Komsuoḡlu, Daniel Koditschek \\ Artificial Intelligence Laboratory, University of Michigan \\ Ann Arbor, MI 48109 \\ \{altendor, ulucs, hkomsuog, kod\}@eecs.umich.edu \\ H. Benjamin Brown Jr. \\ The Robotics Institute, Carnegie Mellon University \\ Pittsburgh, PA 15213 \\ hbb+@cs.cmu.edu \\ Martin Buehler, Ned Moore, Dave McMordie \\ Ambulatory Robotics Laboratory, Dept. of Mech. Engineering, McGill University \\ Montréal, Québec, Canada H2A 2A7 \\ \{buehler, ned, mcmordie\}@cim.mcgill.ca \\ Robert Full \\ Dept. of Integrative Biology, University of California at Berkeley \\ Berkeley, CA 94720 \\ rjfull@socrates.berkeley.edu
}

\begin{abstract}
This paper presents the first evidence that the Spring Loaded Inverted Pendulum (SLIP) may be "anchored" in our recently designed compliant leg hexapod robot, RHex. Experimentally measured RHex center of mass trajectories are fit to the SLIP model and an analysis of the fitting error is performed. The fitting results are corroborated by numerical simulations. The "anchoring" of SLIP dynamics in RHex offers exciting possibilities for hierarchical control of hexapod robots.
\end{abstract}

\section{Introduction}

We have recently reported on a prototype robot that breaks new ground in artificial legged locomotion [1]. Our shoe-box sized, compliant leg hexapod, RHex, travels at speeds better than one body length per second over terrain that few other robots can negotiate at all. RHex origins and construction are grounded in the interplay between biomechanics, controls, and engineering design that we have come to call "functional biomimesis." We aim to articulate broad principles with mathematically precise formulations of biomechanically observed fact and then translate these into specific design practices that make engineering sense. Of course, during a period when the relevant facts are still 
emerging and the appropriate mathematical analysis has yet to be concluded, there is ample scope for intuition guided by existing engineering and biological insight. This paper presents the first empirical evidence that our strategy to use a low degree of freedom mechanism as a "template" for a high degree of freedom task may be relevant and productive. Biomechanics research suggests that the Spring Loaded Inverted Pendulum (SLIP) functions as a sagittal plane template for all animal running [2]. Motivated by the success of Raibert's hoppers [3] that explicitly incorporate a physical SLIP in the working mechanism, we have begun to develop a theory to inform SLIP tuning [4]. We have also reported simulation evidence describing how the two degree of freedom SLIP template may be anchored in a four degree of freedom (all revolute) bipedal running model [5]. Adapting well-characterized methods developed at the UC Berkeley Polypedal lab to explore gait stabilization in animals, we now offer a preliminary characterization of RHex center of mass trajectories respecting which the presumed relevance of the SLIP model can be empirically tested.

\section{The SLIP Template for Legged Runners}

A template [6] is the lowest dimensional model of a robot in an environment that is capable of expressing a specific task as the limit set of a suitably tuned dynamical system involving some controlled (robot) and uncontrolled (environment) degrees of freedom. To "anchor" this low dimensional model in a more physically realistic higher degree of freedom representation of the robot and its environment, we seek controllers whose closed loops result in a low dimensional attracting invariant submanifold on which the restriction dynamics is a copy of the template. Examples of this idea at work in functioning robots include a series of batting machines that anchored a "Raibert vertical" template [7] in a one degree of freedom paddle robot (operating into a two degree of freedom environment) [8] and a three degree of freedom paddle robot (operating into a three degree of freedom environment) [9]. This same idea is used to control a recently reported brachiating robot [10]. In this section we review the manner in which a hierarchical controller can be devised to shape and then exploit the appearance of the SLIP template in morphologically distinct legged machines.

\subsection{Hierarchical Control of a Virtual SLIP Monopod}

The biomechanical evidence for the existence of a SLIP template in human runners $[11,12]$ naturally leads to the possibility of template based controller designs for bipeds. Toward this end, previous work [5] demonstrated an approximate embedding of a SLIP template in a planar 4 DOF leg with ankle, knee and hip joints $(\mathrm{AKH})$, similar in morphology to a human leg.

The hierarchical control of AKH involves defining a virtual leg between the toe and the center of mass (COM) of the system. The joint control torques are then computed using a SLIP template prescribing the ground reaction force (and hence the acceleration of the COM) together with an approximate, virtual work based embedding. In consequence of this hierarchical decomposition, a high level SLIP controller can be used to regulate the speed and hopping height of the overall system. 
Our approach to hierarchical design bears useful comparison to notion of impedance control advanced by Hogan [13] and more recently introduced into the locomotion literature in the more specific form of "Virtual Model Control" by Pratt and colleagues [14]. This framework allows a user to program the robot's task in terms of a reference compliance imposed on a targeted part of the body. The actuator forces and torques are controlled so as to realize the pullback to joint space of the reference co-vector field. A limiting weakness of this otherwise attractive recourse to "programmed stiffness" lies in its ignorance of kinetic energy. The allowable reference models operate by definition in the quasi-static regime, so, for example, running could not lie within the formal scope of the method. Presumably, Pratt's machines function as nicely as they do in fast walking and other apparently dynamical settings because of carefully tuned switching rules that coordinate the pullback to appropriately active and inactive actuators. In contrast, the SLIP template provides an explicitly (hybrid) dynamical specification of the exchange between kinetic and potential energy that accomplishes the task at hand after transients in the many degrees of freedom unrelated to the task have died out. In this paper, we are concerned to find a means of effecting this "collapse" in the RHex mechanics.

\subsection{Hierarchical Control of a Virtual SLIP Hexapod}

In this section, we describe two alternative approaches to hierarchical control for our hexapod. Both appeal to the SLIP template for the prescription of COM forces, but incorporate different anchoring mechanisms.

The hexapod model we consider is a rigid body with six massless legs [1]. Two of the spherical leg freedoms - the radial length and one of the angles - have springs and dampers, whereas the hip angle is torque actuated. Consequently, there are only six actuated joints, and the overall system has six degrees of freedom due to the rigid body.

\subsubsection{Active Control}

In principle, the force and torque on the hexapod rigid body can be determined using the equations of motion of the model [1]. Sufficient conditions for exact embedding of an arbitrary dynamical template can be developed from the invertibility of the dynamics. However, complete input invertibility generally cannot prevail in our system. The morphology of the system as well as the structure and number of the actuators (especially when only one tripod is on the ground), do not yield full control over the six body degrees of freedom.

A simpler planar model, on the other hand, provides an exactly invertible plant, except for co-dimension one and two singularities. The model consists of a three degree of freedom planar rigid body, with six torque actuated massless legs, with the assumption that three or more legs are in contact with the ground during stance.

Preliminary numerical experience with this model suggests that choosing a "reasonable" stance posture affords inverse dynamics controllers that pass transversally through the kinematic singularities and give good SLIP trajectories. Moreover, the planar model is structurally very close to the spatial model. As a consequence, it seems likely that the inverse dynamics anchoring in the 
planar model can be easily extended to yield an approximate embedding of the SLIP template in the spatial hexapod model.

Nevertheless, realizing the active template through inverse dynamics control suffers the traditional problems of all such approaches based on exact cancellations: the presumption of a perfect model; known parameters; and exact high bandwidth state information. Consequently, it is not clear how effectively this exact embedding can be implemented in a physical platform in the face of inevitable actuator, computational, and sensory limitations.

\subsubsection{Passive Control}

An alternative to active control relies on the passive dynamics of the system combined with low-feedback controllers to embed the SLIP template. As the data of Section 3 indicate, such an anchoring seems to be possible even with a very simple open-loop control strategy for certain "sweet spots" in the RHex parameter space. It is still unclear whether this respects the formal "template" paradigm wherein the lower dimensional dynamics actually appears as an attracting invariant dynamical submanifold. However, experimental evidence revealing the template behavior in steady state from various different initial conditions suggests there are, indeed, operating regimes where the system trajectories are attracted to the low dimensional SLIP template dynamics. Further evidence for the SLIP template comes from numerical studies using SimSect - a simulation package developed in C by U. Saranli [15]. SimSect was devised to approximate the behavior of RHex by numerically integrating a set of simplified equations of motion which are expected to govern RHex's hybrid mechanical system. Currently, the same low-level controller as in RHex is implemented in SimSect. This makes SimSect an ideal test-bed for new control designs for RHex. Although an exact correspondence between RHex's and SimSect's parameter space has not yet been established, the simulation results in Sec. 3.5 lend credence to SimSect being a representative numerical approximation to RHex's dynamics.

\section{Finding the SLIP in RHex's Motion}

The central observations about cockroach locomotion that inform the design of the RHex prototype include: (i) that it operates via compliant legs; (ii) that its limb motions appear to be characterized by a strongly stereotypical "clock"; (iii) that it has a sprawled posture to enhance stability; and (iv) that the stabilizing controller must somehow be embedded in the very morphology itself. The force of these observations for RHex are, indeed, directly apparent in the morphology and control approach that we have already reported. However, it is not obvious that we will find SLIP in such a machine.

\subsection{Data Preview}

Before going into the details of experimental procedures and data analysis, we preview force and energy data obtained from experiments with RHex, and compare them to data from cockroach running and to SLIP predictions. Fig. 1 suggests that RHex does anchor a SLIP as does its inspiration, the Blaberus cockroach [16]. Both hexapods use an alternating tripod gait where three 
legs function as a single virtual leg spring. For each single step, an initial deceleration in the fore/aft direction is followed by an acceleration (Fig. 1). Moreover, the relationships between the ground reaction forces and energies
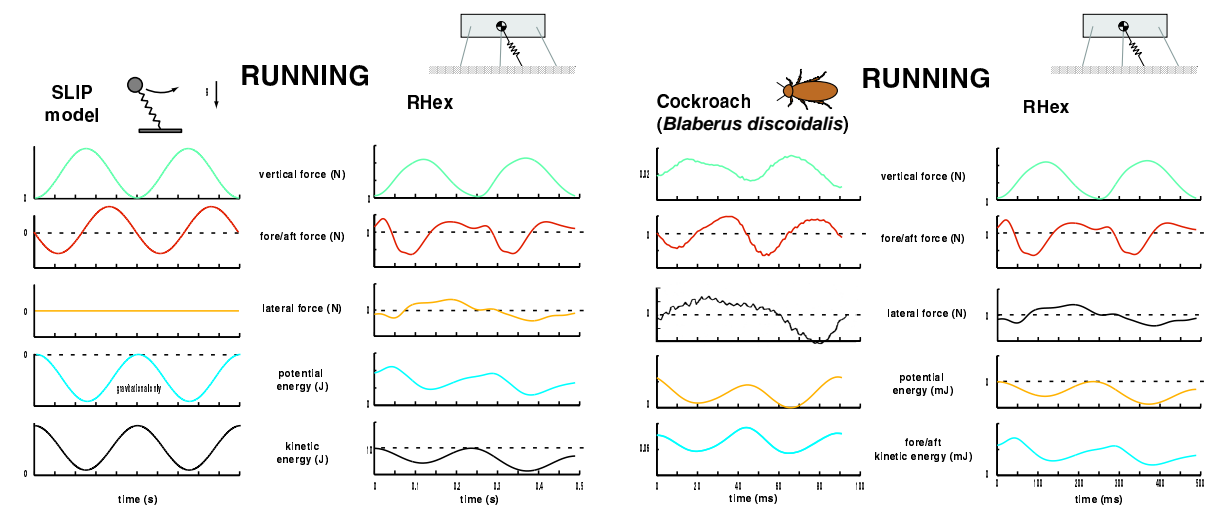

Figure 1. Comparison of sample RHex data with SLIP predictions and with cockroach data.

displayed in this figure may be used qualitatively to compare RHex to the SLIP model. The compelling similarities between the sample RHex and the simulated SLIP stride presented in the figure concern phase-like relationships between the traces. The first two traces indicate that the peak vertical ground reaction force occurs at the transition from negative (deceleration) to positive (acceleration) fore/aft forces. Indeed, comparing the first and third trace, the vertical force peaks at mid-step when the COM is at its lowest height - a necessary and characteristic property of SLIP equilibrium gaits. In contrast, comparing the third and the fourth traces, gravitational potential energy and fore/aft kinetic energy of the COM fluctuate in phase. This "in-phase" relationship between vertical height and horizontal kinetic energy also represents a key feature of SLIP locomotion. Over the last four decades, it has come to be generally adopted within the biomechanics community as the criterion to distinguish walking from running[17], even for animals that do not achieve an aerial phase [18].

\subsection{Data Collection, Experimental Setup and Procedures}

In order to determine if RHex anchors a SLIP, the ground reaction forces produced by RHex during locomotion were measured during ninety-two trials using two six-component force plates (Biomechanics Force Platform, Advanced Mechanical Technology, Inc., Newton, MA). The force and torque signals were amplified (Model SGA, Advanced Mechanical Technology, Inc., Newton, MA) and each channel was recorded at $1000 \mathrm{~Hz}$ by an analog to digital converter (PCI board, National Instruments, Austin, TX) interfaced with a Macintosh. Each trial was also recorded by a high speed video camera (MotionScope PCI 1000, Redlake Imaging, Morgan Hill, CA). The camera was mounted perpendicular 
to the fore/aft axis, approximately two feet above RHex.

In the reported experiments four parameters were varied: The leg type; the ground material; the robot mass; and the forward speed. Two types of legs were used: Delrin legs $(\kappa \approx 4300 N / m)$; and 4-bar linkage composite legs $(\kappa \approx 3100 N / m) .{ }^{1}$ The robot was run on the bare force platform with inelastic collision properties as well as on the force platform covered with an elastic foam mat to reduce slippage. The robot mass was increased by adding weight without changing the COM position. The motion profile utilized by RHex is parametrized by cycle time, sweep angle, leg offset and flight time (for a detailed description of these parameters see [15]). For each forward speed setting a different set of values is assigned to these four parameters.

In our experiments the robot starts walking approximately 2 meter away from the force plates to achieve steady state motion on the plates. While the robot was in contact with the force plates no directional adjustment was made since this would otherwise break the symmetry between the right and left leg motion profiles. Our experiments started off with the slowest forward speed on the bare force plates with Delrin legs. Then the speed was increased in three steps by choosing different cycle times $\left(t_{c} \in\{1.2 s, 0.8 s, 0.53 s, 0.5 s\}\right)$ without changing the physical structure. To reduce bounce and slippage, which was observed especially at high speeds, the surface of the force plates was then covered with an elastic foam mat, and the same speed sweep with the Delrin legs was performed. In the second part of the experimental procedure, new composite legs were used in conjunction with a similar speed sweep on both the bare force plates and the plates covered with the foam mat. Our preliminary observations during the first two trial runs suggested that the COM of the body behaves more like an inverted pendulum (IP) rather than a spring loaded inverted pendulum (SLIP). We reasoned that the leg-body system, which defines an overall lumped spring-mass system, has a much higher natural frequency than the stride frequency achievable by the hip motion. In the third part of the experimental procedure, in order to test this hypothesis, the body mass was increased, effectively decreasing the natural frequency of the spring-mass system. We ran the robot with composite legs at the highest speed setting on the elastic mat. Its mass was increased incrementally from $7.83 \mathrm{~kg}$ to $9.47 \mathrm{~kg}$ to $11.12 \mathrm{~kg}$ to $11.94 \mathrm{~kg}$. In the highest mass regime we observed the transition from IP to SLIP reported below.

\subsection{Data Extraction}

The data plotted in Fig. 2 arise from the summed leg or COM ground reaction forces imparted to the legs by the ground plate while the robot performs an alternating tripod gait. To remove noise from the recorded data, the forces were filtered using a second order Butterworth filter with a cutoff frequency of $50 \mathrm{~Hz}$. The minima of the vertical force data were used to isolate single strides. Since a stride is a complete cycle for all the legs, it contains two steps for each tripod and therefore two minima. Only strides from the middle section

\footnotetext{
${ }^{1}$ These values are only approximate values for the radial stiffness; the spring law is nonlinear and the legs show considerable (non-linear) damping.
} 
of the data for one force platform - where the force data exhibited oscillatory behavior of one predominant frequency and roughly constant amplitude - were selected. Only those trials where the maximum of the power spectrum $P(f)$ occurs at twice the cycle frequency ${ }^{2} f_{c}=1 / T_{c}$ were used, in accord with the criterion established in [19] to distinguish walking from running. ${ }^{3}$ In addition, the ratio of the integrated power spectrum around the maximum to the total integrated power spectrum was required to satisfy $\frac{\int_{f_{c}-\epsilon}^{2 f_{c}+\epsilon} P(f) d f}{\int_{0}^{\infty} P(f) d f}>0.8$, where $\epsilon$ was appropriately chosen to include the global maximum alone. These criteria reduced the number of available trials to be used for SLIP fitting from 92 to 14. Since the force platform is very narrow with respect to the width of RHex

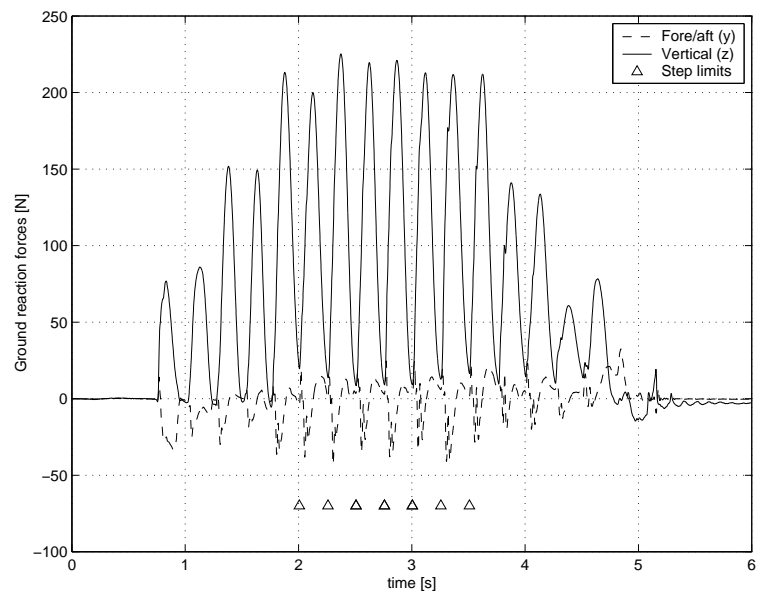

Figure 2. Ground reaction forces for trial no. 6 (composite legs, foam mat, $t_{c}=0.5 \mathrm{~s}, m=11.94 \mathrm{~kg}$ ). The triangles denote the beginning and the end points of steps selected for fitting.

and only those trials for which RHex stayed on the track were recorded, the lateral force data are not used in this investigation. This is also in the spirit of trying to find a sagittal plane SLIP model in RHex's motion.

Unfortunately, the camera's resolution was not good enough to provide integration constants for vertical and fore/aft speeds and positions at the beginning of each stride. Therefore, the initial vertical speed is indirectly obtained from the assumption of periodicity, i. e. that after one stride, the robot returns to its initial height. The fore/aft initial speed is calculated by matching the average velocity over one stride to the average velocity to the average velocity over both force plates. The initial height is assumed to be RHex's neutral height with all legs vertical to the ground $(0.164 \mathrm{~m})$. The initial fore/aft position is irrelevant. The gravitational potential energy and forward kinetic energy for a stride were calculated using these velocities and positions.

\footnotetext{
${ }^{2}$ Remember that during one cycle, two steps are taken.

${ }^{3}$ The criterion mentioned in Sec. 3.1 is not employed here, since it cannot be applied to the raw force data.
} 


\subsection{Data Analysis}

Although the data preview presents time trajectories that compare visually well to the SLIP model, the question arises as to how strong this correspondence really is. The SLIP template imposes a very particular set of relationships those specified by the Lagrangian mechanics of a single point mass prismaticrevolute (i.e., polar coordinate) kinematic chain - between the ground reaction forces, motion of the COM, and system energies. Ruina has pointed out [20] that any convex curve supports in a neighborhood of its bottom point at least one time varying trajectory generated by some SLIP. RHex's COM inevitably rides along a convex curve: we wish to understand whether its actual time trajectory along this curve can be readily generated by some SLIP model.

\subsubsection{A Protocol for Fitting SLIP to RHex's Running Data}

The 14 remaining trials that satisfy the criteria in Sec. 3.3 are now used to test the presence of the SLIP template in RHex. For each of the 136 steps (68 strides) in the 14 trials, a SLIP model is fit. The concept of fitting a SLIP model to those data is not straightforward: since RHex did not have flight phases, the touchdown and lift-off points are not defined. However, one can assume that the region around a vertical minimum can be described by a SLIP stance phase, whereas the corresponding flight phase is replaced by a more complicated mechanical model (e. g. a closed kinematic chain with double support from both tripods; this is sometimes observed in SimSect simulations). The equivalent touchdown and lift-off points (the transition from SLIP to something else) cannot be specified, much less determined, absent a particular model. Hence, a limiting point after which the transition is expected to occur is chosen, namely the point of the zero crossing of the vertical COM force. From the periodicity of the motion, the running behavior of RHex is expected to correspond to a limit cycle of the SLIP model with symmetric trajectories around mid-stance. Therefore the data population for SLIP fitting is chosen to range from a vertical minimum to the next zero crossing of the vertical force.

With the fitting population for a certain step: $z, \dot{z}, \ddot{z}, y, \dot{y}, \ddot{y}^{4}$, the COM acceleration $\ddot{\mathbf{b}}=\left(\begin{array}{ll}\ddot{y} & \ddot{z}\end{array}\right)^{\top}$ and position $\mathbf{b}=\left(\begin{array}{ll}y & z\end{array}\right)^{\top}$ are fitted to a Hooke spring law with unknown spring length $q_{r 0}$ and spring stiffness $\kappa$ :

$$
\frac{\mathbf{b}}{\|\mathbf{b}\|}\left(\kappa_{1}-\kappa_{2}\|\mathbf{b}\|\right)=m(\ddot{\mathbf{b}}-\mathbf{g})
$$

where $\kappa=\kappa_{2}$ and $q_{r 0}=\frac{\kappa_{1}}{\kappa_{2}}$. Fitting to the spring length alleviates the arbitrariness of selecting the equivalent lift-off point, because now the end of the trajectory does not correspond to the lift-off point of the fitted SLIP model. The assessment of the quality of the fit proceeds in two steps:

- First, a SLIP simulation over the same period of time as the data trajectory is run with the values of $\kappa$ and $q_{r 0}$ obtained in the first step. The initial

\footnotetext{
${ }^{4}$ These symbols represent vectors of position, velocity, and acceleration at different times; $z$ gives the co-ordinates in the vertical direction and $y$ gives the co-ordinates in the fore/aft direction. The center of pressure (the pivot point of the virtual slip) is assumed to be below the minimum of the $z$-trajectory.
} 
conditions are taken to be the positions and velocities of the data trajectory at the minimum.

- Second, the resulting SLIP trajectories $z^{\mathrm{SLIP}}, \dot{z}^{\mathrm{SLIP}}, y^{\mathrm{SLIP}}, \dot{y}^{\mathrm{SLIP}}$ are compared to the data trajectories by $L_{1}$ and $L_{2}$ percent errors:

$$
\Delta X_{L_{1}}=100 \frac{\left\|X-X^{\mathrm{SLIP}}\right\|_{1}}{\operatorname{Range}(X)}, \quad \Delta X_{L_{2}}=100 \frac{\left\|X-X^{\mathrm{SLIP}}\right\|_{2}}{\|X\|_{2}} .
$$

Here, $\|X\|_{p}=\left(\int_{t_{0}}^{t_{1}}|X(t)|^{p} d t\right)^{\frac{1}{p}}$ and $X \in\{z, y, \dot{z}, \dot{y}\}$.

In an effort to simplify the assessment of the fitting error, the quality of the fit is reported as a single number - the average $L_{p}$ percent error $\Delta_{L_{p}}=$ $\left(\Delta z_{L_{p}}+\Delta y_{L_{p}}+\Delta \dot{z}_{L_{p}}+\Delta \dot{y}_{L_{p}}\right) / 4$.

As an illustration of the fitting procedure, the worst and the best SLIP fits amongst the 136 steps are presented in the next two figures. The data
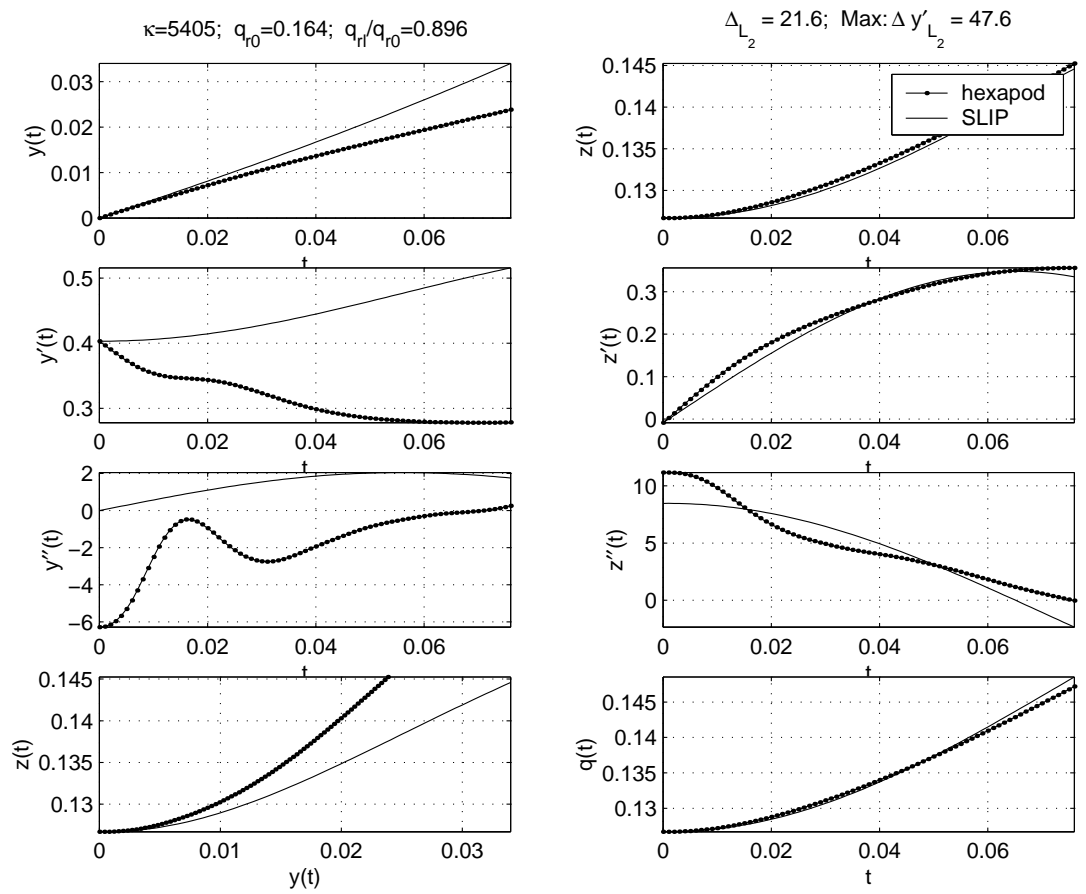

Figure 3. RHex trial no. 2, 4th stride, 2nd minimum (composite legs, foam mat, $t_{c}=0.5 \mathrm{~s}, m=11.12 \mathrm{~kg}$ ) incurs the largest SLIP fitting error of $\Delta_{L_{2}}=21.6 \%$. Dotted lines represent experimental data; solid lines represent SLIP trajectories with fitted values $\kappa$ and $q_{r 0}$.

trajectories of $y(t), \dot{y}(t), \ddot{y}(t), z(y), z(t), \dot{z}(t), \ddot{z}(t)$, and $q(t)=\sqrt{y^{2}(t)+z^{2}(t)}$ (lines with dots) are plotted together with the SLIP predictions computed with the fitted stiffness $\kappa$ and spring length $q_{r 0}$. Above the first left graph, the fitted parameters $\kappa, q_{r 0}$ and the geometric quantity $q_{r l} / q_{r 0}$, where $q_{r l}$ is the leg length 
at the zero crossing of the vertical force, ${ }^{5}$ are printed. Above the first right graph, the total $L_{2}$ error and the maximal $L_{2}$ error are given. The worst SLIP fit with $\Delta_{L_{2}}=21.6 \%$ is shown in Fig. 3, in contrast to $\Delta_{L_{2}}=0.3 \%$ in Fig. 4 .
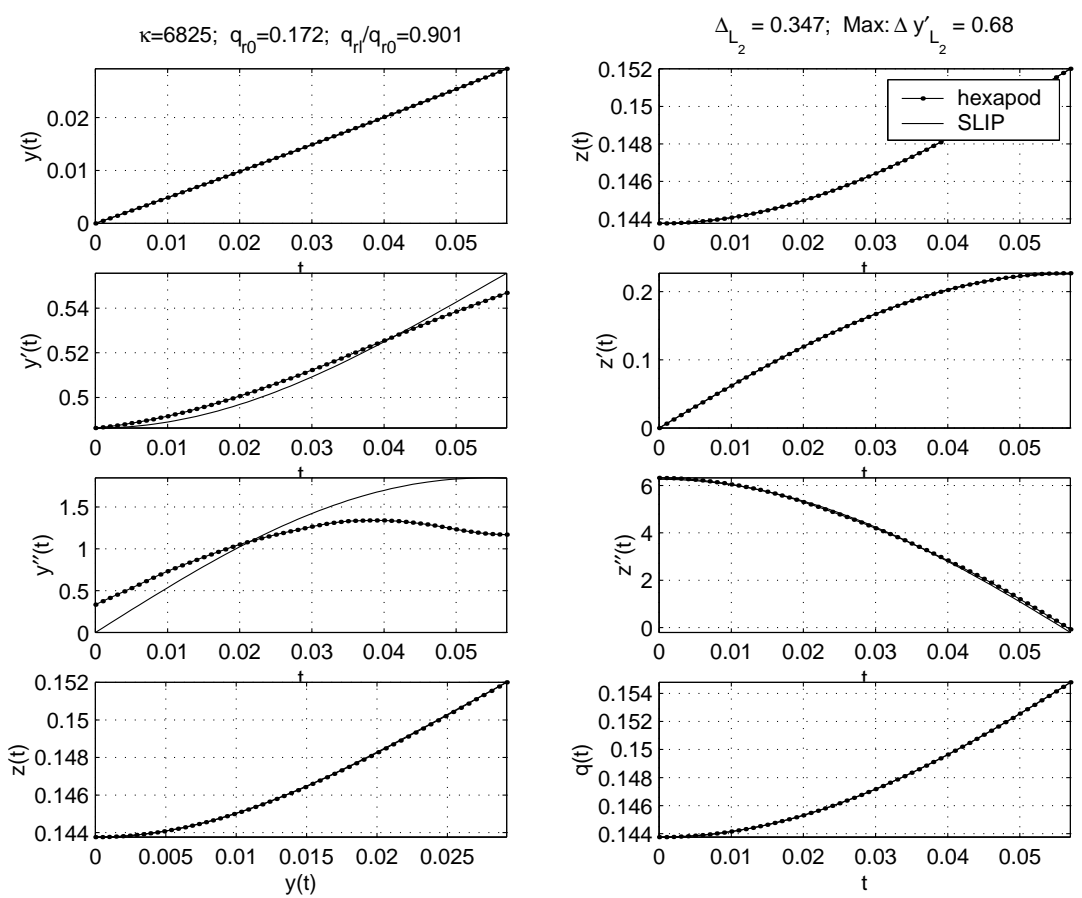

Figure 4. RHex trial no. 10, 5th stride, 1st minimum (composite legs, foam mat, $t_{c}=0.5 \mathrm{~s}, m=11.94 \mathrm{~kg}$ ) incurs the smallest SLIP fitting error of $\Delta_{L_{2}}=$ $0.3 \%$. Dotted lines represent experimental data; solid lines represent SLIP trajectories with fitted values $\kappa$ and $q_{r 0}$.

Then, as an internal consistency check, a simple form of cross validation is performed: the data with data vectors $X \in\{z, y, \dot{z}, \dot{y}\}$ of length $N$ is partitioned into $r=\lfloor N / 20\rfloor$ evenly spaced fitting populations $X_{f i t}^{i}, i \in\{1, \ldots, r\}$ of length $N_{f i t}=\lfloor N / r\rfloor$ and their complements $X_{\text {cross }}^{i}=X \backslash X_{f i t}^{i}$ - the cross validation populations. The fitting procedure is applied to a fitting population $X_{f i t}^{i}$ to yield $\kappa^{i}$ and $q_{r 0}^{i}$, but the quality of the fit is assessed not only on $X_{f i t}^{i}$ but also on the corresponding cross-validation population $X_{\text {cross }}^{i}$ with $\kappa_{f i t}^{i}$ and $q_{r 0, f i t}^{i}$ obtained from $X_{f i t}^{i}$. The fitting errors $\Delta_{L_{p}, f i t}^{i}$ and cross validation errors $\Delta_{L_{p}, \text { cross }}^{i}$ are subsumed under $\Delta_{L_{p}, f i t}=\overline{\Delta_{L_{p} \text { fit }}^{i}}, \Delta_{L_{p}, \text { cross }}=\overline{\Delta_{L_{p} \text { cross }}^{i}}$, and the standard deviations $\operatorname{std}\left(\Delta_{L_{p}, f i t}^{i}\right)$ and $\operatorname{std}\left(\Delta_{L_{p}, \text { cross }}^{i}\right)$.

In addition to the $L_{1}$ and $L_{2}$ errors that compare the experimental data to fitted SLIP predictions, experimental measurement errors introduce noise

\footnotetext{
${ }^{5}$ The ratio $q_{r l} / q_{r 0}$ is an indicator of how well the part of the data population chosen for fitting approximates the whole SLIP stance trajectory.
} 
into the data. For the fitting procedure to be meaningful, one would like the noise floor $\delta X / \operatorname{Range}(X)$ to be well below the $L_{1}$ fitting errors. The noise floor comes from two sources: the measurement error of the ground reaction forces $F_{\text {ground }}$ with $\delta F_{\text {ground }} \approx 0.01 F_{\text {ground }}$, and the uncertainties of the integration constants for the velocity and position trajectories. The resulting noise floors for the $z, y, \dot{z}, \dot{y}$ trajectories are then averaged over one stride.

\subsubsection{Evidence for SLIP in RHex Running}

The fitting protocol outlined in the previous section is now applied to all 136 steps. For the sake of brevity, we refrain from listing the fitted parameters and the fitting errors for each step. Instead, average values over all steps are calculated together with the standard deviation. The mean fitted stiffness is $\kappa=(6100 \pm 940) \mathrm{N} / \mathrm{m}$, and the mean fitted relaxed spring length is $q_{r 0}=$ $(0.171 \pm 0.007) m$. The results of the error analysis are listed in Table 1. By

\begin{tabular}{|l||c|c|c|c|}
\hline$(\%)$ & $\Delta_{L_{p}}$ & $\Delta_{L_{p}, \text { fit }} / \Delta_{L_{p}, \text { cross }}$ & $\Delta_{L_{p}, \text { cross }}$ & std $\left(\Delta_{L_{p}, \text { cross }}\right)$ \\
\hline \hline$L_{2}$ & $0.069 \pm 0.054$ & $0.985 \pm 0.013$ & $0.069 \pm 0.054$ & $0.001 \pm 0.001$ \\
\hline \hline$L_{1}$ & $0.258 \pm 0.197$ & $1.002 \pm 0.012$ & $0.259 \pm 0.199$ & $0.004 \pm 0.006$ \\
\hline \hline \hline$(\%)$ & $\Delta y_{L_{p}}$ & $\Delta z_{L_{p}}$ & $\Delta \dot{y}_{L_{p}}$ & $\Delta \dot{z}_{L_{p}}$ \\
\hline \hline$L_{2}$ & $0.094 \pm 0.079$ & $0.001 \pm 0.001$ & $0.146 \pm 0.117$ & $0.036 \pm 0.033$ \\
\hline \hline$L_{1}$ & $0.041 \pm 0.036$ & $0.010 \pm 0.010$ & $0.96 \pm 0.78$ & $0.020 \pm 0.018$ \\
\hline Noise floor & 0.009 & 0.003 & 0.38 & 0.009 \\
\hline
\end{tabular}

Table 1. Error analysis of SLIP fitting to 136 steps.

comparing the last two rows it can be seen that the noise floor is well below the mean $L_{1}$ errors, as required for a meaningful fitting procedure. Also, the average $\Delta_{L_{2}}$ error of $\approx 7 \%$ seems to be remarkable for a mechanical device that a priori bears to resemblance to a SLIP model.

All of the selected 14 trials come from experiments with RHex operated in the highest speed setting (i. e. with a cycle time of $0.5 \mathrm{~s}$ ). The other settings for this speed are: sweep angle $=51^{\circ}$, leg offset $=0^{\circ}$, and flight time $=0.2 s$. The flight time is less than half of the cycle time, which means that the controller already dictates a gait with periods of double support from both tripods. In all 14 trials the new composite legs with low radial stiffness and the elastic foam mats to reduce slippage were used. Moreover, the masses for the selected trials were mainly in the high mass regime: 8 trials with $m=11.94 \mathrm{~kg}, 5$ trials with $m=11.12 \mathrm{~kg}$, and 1 trial with $m=9.47 \mathrm{~kg}$. This can be explained by comparing the natural period of oscillation $T_{\kappa, m}$ of the (leg-) spring/mass system to the time period of a stance phase (sweep time) $T_{S}$, as already mentioned in Sec. 3.2. SLIP predicts $T_{\kappa, m}=2 T_{S}$, where $T_{\kappa, m} \sim \sqrt{m / \kappa}$. For slow speed settings and low masses, $T_{S}$ is much larger than $T_{\kappa, m}$; by increasing the speed ${ }^{6}$ and mass one makes $T_{S}$ and $T_{\kappa, m}$ converge.

\footnotetext{
${ }^{6}$ Higher speed settings have lower sweep times.
} 


\subsection{Supporting Numerical Study}

\subsubsection{SLIP Fitting in SimSect}

The previous sections demonstrates that the SLIP model provides a good approximation to the "stance" dynamics of RHex. In this section, we describe a parallel numerical investigation of SimSect simulations to determine the "sweet spots" wherein the hexapod might actually be presumed to anchor the SLIP. Specifically, we show that SLIP-like behavior of the mechanical system modeled by SimSect occurs in specific ranges of SimSect's parameter space. This parameter space can be categorized into physical parameters like total mass, moments of inertia, etc, initial conditions, and control parameters like sweep angle, cycle time, leg offset, and flight time. ${ }^{7}$ The physical parameters are chosen to match those of RHex; this is straightforward for e. g. the total mass; the parameters modeling a leg, i. e. radial stiffness, radial damping, etc, however, can only be guessed, since RHex's legs have complicated non-linear compliances and dampings, whereas in SimSect the legs are modeled by a linear spring law with viscous damping and an exponential saturation (see [15]). Hence, the physical parameter space of SimSect where good SLIP fits can be obtained does not necessarily match RHex's parameter space.

The RHex trials from Sec. 3.2 do not have flight phases. Although flight phases can be obtained in SimSect, one would like to be able to fit a SLIP model to any SimSect simulations. For the RHex data, a somewhat arbitrary criterion to determine which part of the trajectory to use for fitting was imposed. In order to compare the SLIP fitting results from SimSect to those from RHex experiments, the same part of the decompression trajectory as in Sec. 3.4.1 is used for fitting. ${ }^{8}$ Only those runs where exactly 3 legs of the same tripod are on the ground at the vertical minimum were considered to be acceptable; this was inspired by the assumption that the jointly controlled three legs, which constitute a tripod, can be thought of as a virtual SLIP leg. As an additional filter only those simulations which exhibit periodic behavior after a certain amount of time are used for fitting to the SLIP model.

The simulations are run for a fixed set of physical parameters and initial conditions, whereas the control parameters sweep angle, cycle time, and leg offset are varied in certain ranges described below. The flight time is chosen to $\mathrm{be} \approx 0.4$ the cycle time in order to match the highest speed setting for RHex. With force, velocity, and position data from SimSect simulations, the fitting procedure is carried out as in Sec. 3.4.1.

\subsubsection{Fitting Results}

Although SimSect is modeled with many simplifications respecting the actual robot, RHex's main dynamical features are believed to be incorporated in SimSect. Hence the SimSect simulations were run in parameter regimes that include the high mass, high speed regimes of RHex where good SLIP

\footnotetext{
${ }^{7}$ For a detailed description of SimSect's parameter space, see [15].

${ }^{8}$ In SimSect, information about which legs are on the ground and which are not is available. A detailed investigation of the quality of the SLIP fitting as a function of footfall patterns will be the subject of a future report.
} 
fits could be obtained. In particular, with total mass $m=11.9 \mathrm{~kg}$, sweep angle $=0.44-0.76 \mathrm{rad}$, cycle time $=0.42-0.54 \mathrm{~s}$, leg offset $=0-(-0.15) \mathrm{rad}$, and individual leg stiffnesses $\kappa \approx 2700 N / m$, SLIP like behavior could be found in SimSect, too. This is demonstrated in Fig. 5, which shows - on the left side a histogram of the number of simulations from the above listed parameter range with respect to the average $L_{2}$ error $\Delta_{L_{2}}$. The total number of simulations is reported above the histogram. For comparison, a two-parameter exponential distribution is fit to the histogram bins. On the right side a graph shows the average $L_{2}$ error $\Delta_{L_{2}}$ as a function of two of the four control parameters: cycle time and sweep angle. The flight time is kept proportional to the cycle time and only a small dependence of $\Delta_{L_{2}}$ on the leg offset was observed. Instead of a scatter plot, a quadratic surface is fit to the data, and the spread of the data is characterized by its second moment around the surface, which is represented by vertical bars at the corners of the surface.
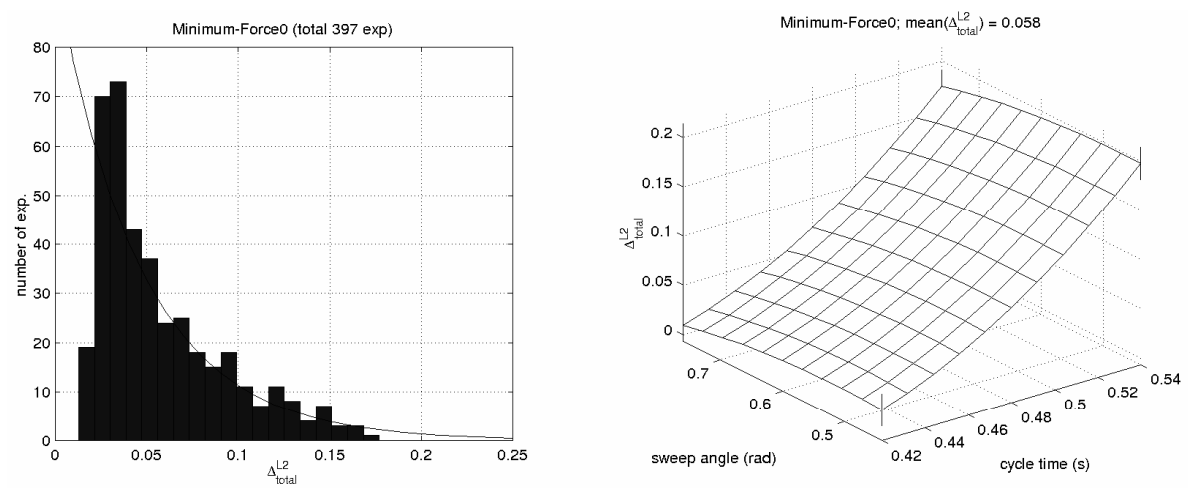

Figure 5. Average errors $\Delta_{L_{2}}$ for SimSect SLIP fits. The left side shows a histogram of the number of simulations; the right side shows a maps of $\Delta_{L_{2}}$ as a function of the (reduced) control parameter space.

For all SimSect simulations the ratio of the average fitting to the average cross validation error $\Delta_{L_{2}, \text { fit }} / \Delta_{L_{2}, \text { cross }}$ did not deviate by more than $10 \%$ from unity, thus providing a successful self-consistency check for our fitting procedure. The results in this section, in particular the low average fitting errors of $\approx 6 \%$ in Fig. 5 lend strong support to the assumption that the SLIP template may be anchored in SimSect's dynamics.

\section{Conclusion: Implications for More Autonomous Con- trol of RHex}

Hierarchy promotes the use of few parameters to control complex systems with many degrees of freedom. In this light, as we understand matters, the emergence of an anchored SLIP in RHex is most fortunate. The pogo-stick can function as a useful control guide in developing more complex autonomous locomotion behaviors such as registration via visual servoing, local exploration via visual odometry, obstacle avoidance, and, eventually, global mapping and 
localization. In the longer term, we propose to work with the anchored SLIP in RHex in analogy to the manner in which the simple two-bead template has been exploited in juggling. Namely, as we shape behavior via manipulation of gains-in-the-loop [21], we hope to develop a formal programming language with semantics in the world of dynamical attractors [22].

\section{Acknowledgements}

This work is supported by DARPA/SPAWAR under contract N66001-00-C8026. We thank Rodger Kram and Claire Farley for the use of the force platform and Irv Scher for collaboration at an early stage of this project.

\section{References}

[1] Saranli U, Buehler M and Koditschek D E 2000 Design, Modeling and Preliminary Control of a Compliant Hexapod Robot. Proc. IEEE Int. Conf. Rob. Aut. $3: 2589-2596$.

[2] Blickhan R and Full R 1993 Similarity in multilegged locomotion: Bouncing like a monopode. J. J. Comp. Physiol. A 173, 509-517.

[3] Raibert M 1986 Dynamic Robots that Balance, MIT Press, Cambridge.

[4] Schwind W J and Koditschek D E 2000 Approximating the Stance Map of a 2 DOF Monoped Runner. Journal of Nonlinear Science.

[5] Saranli U, Schwind W J, and Koditschek D E May 1998 Toward the Control of Multi-Jointed, Monoped Runner. IEEE Int. Conf. on Rob. and Aut. Leuven, Belgium pp 2676-2682.

[6] Full R J and Koditschek D E 1999 Templates and Anchors: Neuromechanical Hypotheses of Legged Locomotion on Land. J. Exp. Bio. 202:3325-3332.

[7] Bühler M, Koditschek D E, and Kindlmann P J 1990 Planning and Control of a Juggling Robot. In: Hayward V, Khatib O(eds) 1990 Experimental Robotics I Springer-Verlag, pp 35-73.

[8] Bühler M, Koditschek D E, and Kindlmann P J 1990 A Family of Robot Control Strategies for Intermittent Dynamical Environments. IEEE Control Systems Magazine 10(2):16-22.

[9] Rizzi A A, Whitcomb L L, and Koditschek D E 1992 Distributed Real-Time Control of a Spatial Robot Juggler. IEEE Computer 25(5):12-24.

[10] Nakanishi J, Fukuda T, and Koditschek D E 2000 A Brachiating Robot Controller. IEEE Trans. Rob. Aut. 16(2):109-123.

[11] Schwind W J 1998 Spring Loaded Inverted Pendulum Running: A Plant Model. $\mathrm{PhD}$ thesis, University of Michigan.

[12] Full R J and Farley C T 2000 Musculoskeletal Dynamics in Rhythmic Systems: A Comparative Approach to Legged Locomotion. In: Winter, Crago (eds) Biomechanics \&6 Neural Control of Posture 86 Movement Springer Verlag, New York, pp 192-205.

[13] Hogan N Mar 1985 Impedance Control: An Approach to Manipulation. ASME Journal of Dynamic Systems, Measurement, and Control 107:1-7.

[14] Pratt J and Pratt G May 1998 Intuitive Control of a Planar Bipedal Walking Robot ICRA Leuven, Belgium pp 2014-2021.

[15] Saranli U 2000 SimSect Hybrid Dynamical Simulation Environment. University of Michigan Technical Report CSE-TR-437-00. 
[16] Full R J and Tu M S 1990 Mechanics of six-legged runners J. Exp. Biol. 148:129146.

[17] Cavagna G A, Saibene F P, and Margaria, R 1964 Mechanical Work in Running. J. Appl. Physiol. London, 19(2):249-256.

[18] McMahon T A 1985 Role of compliance in mammalian running gaits J. Exp. Biol. 115:263-282.

[19] Alexander R McN 1992 A Model of Bipedal Locomotion on Compliant Legs Phil. Trans.: Biol. Sc. 338(1284):189-198.

[20] Ruina A, personal communication.

[21] Burridge R R, Rizzi A A, and Koditschek D E 1999 Sequential Composition of Dynamically Dexterous Robot Behaviors. Int. J. Rob. Res. 18(6):534 - 555.

[22] Klavins E and Koditschek D E 2000 A formalism for the composition of concurrent robot behaviors. Proc. IEEE Conf. Rob. and Aut. 4:3395-3402. 